\title{
Pathogenesis of Thrombocytopenia in Chronic HCV Infection: A Review
}

\author{
Sarah Rawi* and George Y Wu \\ Department of Medicine, Division of Gastroenterology-Hepatology, University of Connecticut Health Center, Farmington, CT, USA
}

\begin{abstract}
A large proportion of patients with chronic hepatitis $C$ have associated thrombocytopenia (TCP). Due to bleeding risks, $\mathrm{TCP}$, when severe, can limit diagnostic and therapeutic procedures, treatments, and increases risk of complications, especially excessive bleeding. It is important to understand the mechanisms that cause TCP in order to manage it. In general, TCP can be due to increased destruction or decreased production. Proposed mechanisms of increased destruction include autoantibodies to platelets and hypersplenism with sequestration. Proposed mechanisms of decreased production include virus-induced bone marrow suppression and decreased TPO production. Autoantibodies directed against platelet surface antigens have demonstrated an inverse correlation with platelet counts. Hypersplenism with sequestration involves the interaction of portal hypertension, splenomegaly, and platelet destruction. Decreased production mechanisms involve appropriate and inappropriate levels of TPO secretion. There is limited evidence to support viral-induced bone marrow suppression. In contrast, there is strong evidence to support low levels of TPO in liver failure as a major cause of TCP. TPO-agonists, specifically eltrombopag, have been shown in hepatitis $C$ patients to increase platelet counts without reducing portal hypertension or splenomegaly. We conclude that TCP in hepatitis C virusinduced liver disease is often multifactorial, but an understanding of the mechanisms can lead to judicious use of new drugs for treatment.
\end{abstract}

Citation of this article: Rawi S, Wu GY. Pathogenesis of thrombocytopenia in chronic HCV infection: A review. J Clin Transl Hepatol 2020;8(2):184-191. doi: 10.14218/JCTH.2020.00007.

\section{Introduction}

Thrombocytopenia (TCP) is most often defined by platelet (PIt) counts less than $150 \times 10^{9} / \mathrm{L} .{ }^{1}$ The average PIt survival time has been reported to range from 7.5 to 9.5 days. $^{2}$ It has been estimated that at least 71 million individuals in the world

Keywords: Thrombocytopenia; Chronic hepatitis; Autoantibodies; Hypersplenism; Thrombopoietin.

Abbreviations: $\mathrm{HCV}$, hepatitis $\mathrm{C}$ virus; IFN, interferon; ITP, idiopathic thrombocytopenia purpura; PAIgG, platelet-associated immunoglobulin G complexes; PIt, platelet; TCP, thrombocytopenia; TIPS, transjugular intrahepatic portosystemic shunt; TPO, thrombopoietin.

Received: 30 January 2020; Revised: 25 March 2020; Accepted: 31 March 2020 *Correspondence to: Sarah Rawi, Department of Medicine, University of Connecticut Health Center, 263 Farmington Ave, Farmington, CT 06032, USA. Tel: +1-858-692-2372, E-mail: rawi@uchc.edu and 2.7-3.9 million people in the USA have chronic hepatitis $\mathrm{C}$ infection. ${ }^{3-4}$ From $0.16 \%$ up to $76 \%$ of these patients have associated TCP. $^{5}$ Populations with high percentages of advanced cirrhosis reportedly have increased prevalence of TCP. TCP is important to recognize because it can increase the risk of complications during invasive diagnostic or therapeutic procedures, need for treatment with interferon (IFN), and in the management of patients on orthotropic liver transplantation waiting lists. It can be a risk factor for bleeding esophageal varices, in chemotherapy for solid tumors or hematological malignancies, and surgery. ${ }^{6,7}$ Bleeding risk increases as PIt levels decrease below $50 \times 10^{9} / \mathrm{L}$, with major bleeding associated at levels below $10 \times 10^{9} / \mathrm{L}$. However, PIt counts alone do not always reflect bleeding, as other factors such as Plt function, the presence of anti-Plt antibodies, and levels of coagulation factors may also be involved. ${ }^{8}$

In order to better understand how to treat or manage TCP, it is essential to understand the pathophysiological mechanisms that cause it. Such knowledge can lead to selection of appropriate therapy.

TCP can be caused by increased destruction (including increased storage) of Plts or decreased production of Plts (Fig. 1). ${ }^{1}$ Conditions that cause increased destruction of Plts include autoimmune responses to antibodies against Plts, idiopathic TCP purpura (ITP), and hypersplenism. Hypersplenism is characterized as splenomegaly and TCP. ${ }^{9}$ TCP in this case can be due to increased storage of Plts, with PIt sequestration and destruction of Plts by phagocytosis, as well as autoimmune responses. In this paper, the term 'destructionsequestration' is used to encompass the mechanisms behind TCP caused by hypersplenism. This is an important distinction because PIt 'destruction', 'degradation', and 'sequestration' may have different mechanisms of action, some of which have not been proven, but are often used interchangeably in other articles/studies.

On the other hand, conditions that cause decreased production of Plts include virus-induced bone marrow suppression and decreased thrombopoietin (TPO) production. Not infrequently, several mechanisms may coexist. The purpose of this review is to present the current state of knowledge regarding the pathogenesis, diagnosis and treatment of TCP in patients with hepatitis $\mathrm{C}$ virus (HCV).

\section{Increased destruction}

\section{Auto-antibodies}

The relationship between autoimmune disease and TCP in $\mathrm{HCV}$ has been studied by analysis of anti-tissue antibodies (antinuclear and anti-smooth muscle antibodies), 


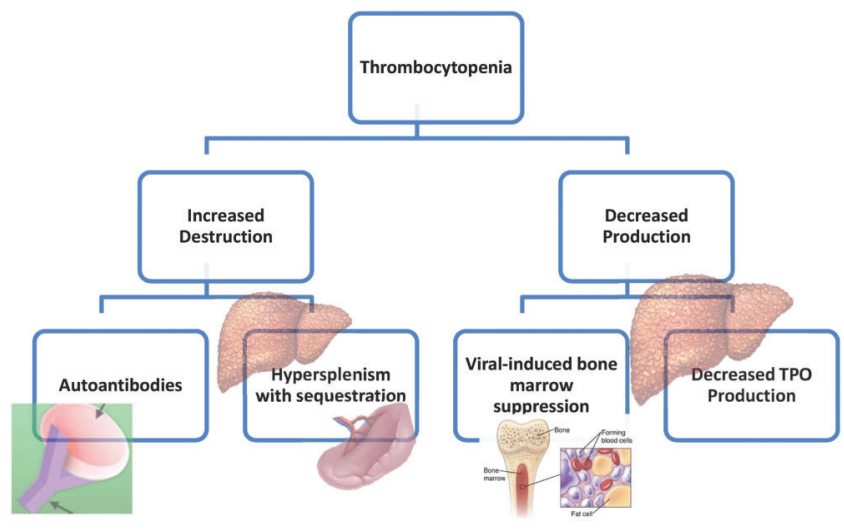

Fig. 1. Pathophysiologic mechanisms of thrombocytopenia.

cryoglobulinemia, rheumatoid factor, and kidney and microsomal antibodies. ${ }^{10,11}$ Autoantibodies directed against PIt surface antigens have been identified. These antibodies are thought to become Plt-associated immunoglobulin G complexes (PAIgG) recognized by macrophages in the spleen or liver. ${ }^{11}$ Once recognized by macrophages, premature destruction of the PIts occurs by phagocytosis within the reticuloendothelial system. ${ }^{11-14}$ Up to $40 \%$ of chronic hepatitis C patients have autoantibodies ${ }^{10}$ and up to $30 \%$ of patients with chronic ITP have been found to have chronic HCV. ${ }^{15,16}$

Nagamine et al. ${ }^{17}$ compared chronic hepatitis $C$ patients with chronic hepatitis $B$ patients and controls, with the purpose of determining the relationship between PAIgG and PIt counts. Up to $88 \%$ of patients with chronic HCV have had high levels of PAIgG compared to their controls $(p<0.01)$, with an inverse correlation of Plt levels $(p<0.05) .11,17$ The PAIgG levels increased with histological progression of hepatitis $C$, further supporting their endpoint. A weakness of the study is that at the time the study was performed, there was no assay for detection of PIt-specific autoantibodies; instead, total PAIgG levels were used. On the other hand, the PAIgG levels did not correlate with serum IgG levels. The study also suffered from a small patient population ( $n<100$ per group) and a lack of measurement of specific autoantibodies.

In another study, two groups of patients, one with low PIt counts $\left(<150 \times 10^{9} / \mathrm{L}\right)$ and the other with normal PIt counts, were studied to determine differences in levels before and after IFN therapy. ${ }^{18}$ In addition, that study measured levels of PAIgG in each subgroup to determine the relationship between TCP and an autoimmune response. Patients with cirrhosis, splenomegaly, chronic alcohol use and other autoimmune chronic liver diseases were excluded. At baseline, PAIgG levels were $181 \%$ higher in the TCP group (197.3 $\left.\pm 130.2 \mathrm{ng} / 10^{7} \mathrm{PIt}\right)$ compared to the non-TCP group (70.9 $\left.\pm 31.1 \mathrm{ng} / 10^{7} \mathrm{PIt}\right)(p<0.01)$. Although high levels of PAIgG were found in the TCP group, the study was not designed specifically to detect the relationship between immune dysfunction and TCP in HCV. PAIgG levels were lower in patients observed after IFN treatment. Unfortunately, exact numbers/ percentage decreases were not studied and/or reported. These data are required to establish the existence of HCVinduced autoimmune responses.

HCV may contribute to or trigger the development of ITP, mediated in particular by circulating immune complexes. Pockros et al. ${ }^{16}$ studied 3440 new HCV cases to determine the prevalence of new HCV-ITP cases. In that study, HCV-ITP was diagnosed by the following criteria: the diagnosis of HCV either before or concurrent with documentation of TCP, TCP out of proportion to the severity of the liver disease, a positive anti-Plt antibody test, and a response to agents known to be effective in the treatment of ITP (e.g., corticosteroids, cyclophosphamide) in patients who required therapy (six of the seven patients). Within a 54-month interval, there were seven new HCV-ITP cases associated with antibodies against GPIIb/IIIa. All of these patients were treated with steroids initially and only two responded by return to normal PIt counts. Three required steroids and intravenous immunoglobulin, and two required cyclophosphamide and splenectomy. Even with these treatments, the PIt counts were still low and the highest count was only around $50 \times 10^{9} / \mathrm{L}$. Statistical analyses showed that those differences in HCV-ITP prevalence were highly significant. However, there were no data on treatment of the virus itself to determine its effect on PIt counts and antibody levels. It is important to note that all patients in the study were also positive for other autoimmune factors, such as antinuclear antibodies, anti-smooth muscle antibodies, or cryoglobulins, which are confounding factors. The study also had a very small patient population, which reduces its external validity. Overall, it is difficult to justify the conclusions of the study.

Honma et al. ${ }^{19}$ prospectively studied 187 patients who were treated with direct-acting antivirals. PAIgG levels as well as Plt counts were measured prior to treatment and post-treatment. Among the patients, $91.4 \%$ had elevated PAIgG levels before the study. However, only $18.2 \%$ of the patients had TCP, which the study defined as $<100 \times 10^{9} / \mathrm{L}$. Because of this low cutoff value, the number of individuals with TCP is likely to have been an underestimate. Among the 34 thrombocytopenic patients, $97.1 \%$ had elevated PAIgG levels. That study claimed that 12 and 24 weeks after end of treatment with direct-acting antivirals, there was a statistically significant increase in PIt counts and decrease in PAIgG levels, with the largest change in the individuals who had the lowest baseline PIt count and highest baseline PAIgG level ( $p=0.021$ and $p=0.002$, respectively). Their data showed a statistically significant negative correlation between PIt count and PAIgG levels before and after directacting antivirals $(p<0.001)$, which supports an autoimmune role in Plt destruction. However, larger studies are needed to confirm this conclusion.

Other studies or components of studies dispute the autoimmune theory of PIt destruction. By measuring PAIgG levels specifically, Pereira et al. ${ }^{12}$ studied 35 patients with chronic liver disease to determine whether anti-PIt antibodies were involved in the development of TCP. Twenty-three of the thirty-six patients (64\%) had anti-GP IIb/IIIA or GpIb antibodies found by using a specific glycoprotein immunoassay of IgG bound to those complexes. The specific antibody levels correlated with elevated levels of PAIgG. However, elevated levels of PAIgG were not inversely correlated to Plt counts. The data supported autoimmune destruction of Plts and not viral suppression. Autoimmune destruction is mediated by the reticuloendothelial system, whereby phagocytosis of Plt immune complexes is mediated by Fc receptors.

In another study, 78 patients with chronic HCV but without other autoimmune diseases were studied to determine the relationship to PAIgG formation and to compare PAIgG levels before and after IFN treatment. ${ }^{20}$ Of these patients, $83.3 \%$ had elevated PAIgG titers (median titer of $400 \mathrm{ng} / 10^{7} \mathrm{PIt}$ ) 
compared to the upper normal value of $100 \mathrm{ng} / 10^{7} \mathrm{plt}$. Of these patients, 23 underwent IFN treatment. Sixteen patients $(69.5 \%)$ were responders (sustained virologic response) and were found to have significantly higher PAIgG titers $(622 \mathrm{ng} /$ $10^{7} \mathrm{plt}$ ) compared to non-responders $\left(283 \mathrm{ng} / 10^{7} \mathrm{plt}\right.$; $p=0.002)$. The Plt counts decreased in the responder group from $195 \times 10^{9} / \mathrm{L}$ to $146 \times 10^{9} / \mathrm{L}(p=0.0001) .{ }^{20}$ That study also measured PAIgG levels before and after treatment. Strengths of the study included exclusion of other autoimmune disorders and assessment of the effect of eradication of HCV on PAIgG levels. These data argue against HCVinduced autoimmune antibodies leading to Plt destruction because the PAIgG titers increased while Plt counts decreased after eradication of HCV. However, the study could have been improved by evaluating specific effects of IFN on PIt counts after treatment.

Overall, autoimmune antibodies may be a contributory factor to TCP but there is not enough evidence to suggest it to be a sole cause of TCP in most cases of chronic HCV.

\section{Hypersplenism with sequestration}

As portal hypertension develops, the spleen enlarges and resistance to portal flow increases, causing redistribution/ pooling of PIts in the spleen and therefore decreased PIts in circulation (sequestration). ${ }^{21,22}$ The incidence of splenomegaly in cirrhosis has been reported to range from $36-92 \%$, while the incidence of hypersplenism in cirrhosis ranged from $11-55 \%{ }^{23}$ Fig. 2 shows the relationships between liver cirrhosis, TCP, and hypersplenism.

By application of radiolabeled Plts in patients with splenomegaly, it has been shown that the primary site of sequestration is the spleen rather than the liver. In one study, there was a $34 \%$ increase in Plt sequestration in patients with splenomegaly compared to those without. The mean PIt survival time was also studied and showed a $2.2 \pm 0.2$ day decrease in survival time in patients with splenomegaly compared to their normal controls. ${ }^{24,25}$ However, rate of sequestration was not studied, and although the term 'PIt destruction' was used, the evidence for this was not clear. There is evidence that PIt destruction-sequestration caused by hypersplenism is an autoimmune process. In this case, Plts are bound by anti-PIt

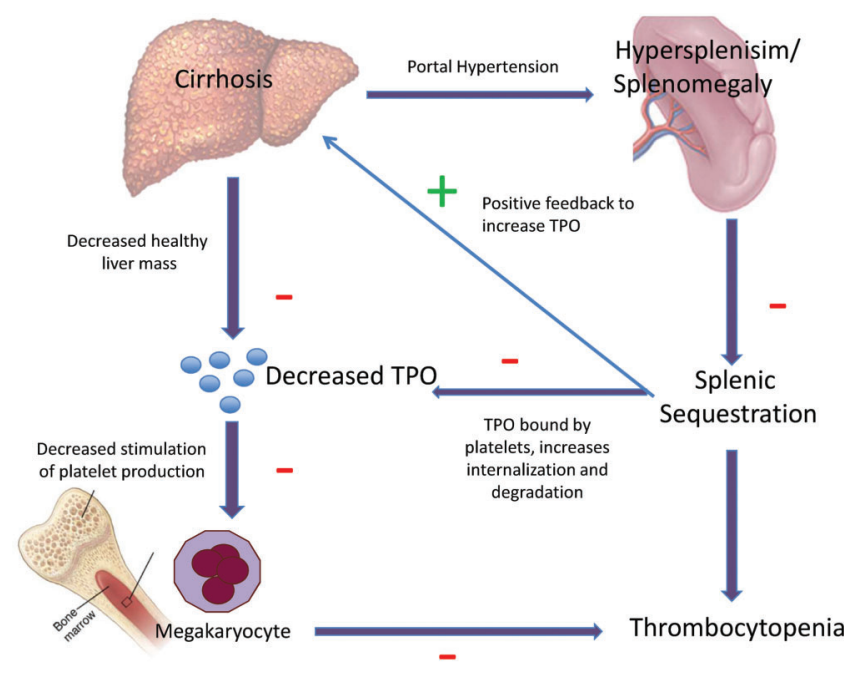

Fig. 2. Relationships between cirrhosis and thrombocytopenia. antibodies and destroyed by macrophages. ${ }^{23,26}$ In the study by Sekiguchi et al., ${ }^{26} 24$ patients with HCV cirrhosis were compared to $17 \mathrm{HCV}$ asplenic cirrhotic patients and 21 non$\mathrm{HCV}$ cirrhotic patients to determine the relationship between spleen size, PAIgG, and PIt counts. Individuals with HCV and splenomegaly had a $61 \%$ increase in average PAIgG titers $(247.9 \pm 197)$ compared to those splenectomized (125.6 \pm 87.8 ) and non-HCV cirrhosis patients (152.4 \pm 127.4 ). There was an inverse correlation between PAIgG and PIt counts. When titers were as high as $400 \mathrm{ng} / 10^{7}$ cells, the PIt counts decreased to below $60 \times 10^{9} / \mathrm{L}$. Furthermore, in patients who were splenectomized, there was no association with PAIgG and PIt counts, and there was a decreased T cell response reflected in decreased CD4/CD8 ratios. Thus, there appeared to be a relationship between splenomegaly and an autoimmune form of destruction-sequestration, resulting in decreased circulation of Plts. Overall, the data from that study was convincing for this relationship when comparing three separate groups. However, it was a retrospective study with the inherent weaknesses of such.

In another study, 209 patients with chronic viral hepatitis were investigated, of which 85 patients had splenomegaly and 124 had a normal spleen size. HCV was the etiology in $93 \%$ of the cases. Of the patients with splenomegaly, $71 \%$ had TCP. As expected, there was an inverse relationship between spleen size and PIt count, along with a direct relationship between spleen size and portal vein diameter. On the other hand, there was no correlation between liver fibrosis and spleen size, which was unexpected as progression to cirrhosis and portal hypertension were expected to cause splenomegaly. Moreover, in that study, as fibrosis increased, the Plt count decreased $(p<0.001)$. In the subgroup of patients with grades 3 and 4 fibrosis without splenomegaly, $76 \%$ had TCP and the median Plt count was $108-126 \times 10^{9} / \mathrm{L}^{27}$ The study had appropriate exclusion criteria and a sufficient number of patients studied, with overall equal demographics. However, the study only had $34 \%$ of patients with grades 3 or 4 fibrosis, which could have falsely lowered their results. Overall, this study demonstrated that splenomegaly leading to sequestration could not be the sole mechanism of TCP.

Furthermore, studies have shown that there was no significant difference in Plt counts between patients with and without splenomegaly. In patients who were already thrombocytopenic, the Plt counts were significantly different in those patients with enlarged spleens compared to without (mean Plt: $77 \pm 26 \times 10^{9}$ vs. $115 \pm 30 \times 10^{9}$ cells $/ L$ $(p<0.0001)) .{ }^{27}$ Splenomegaly may contribute to TCP but is often not sufficient to cause TCP.

To examine the relationship between portal hypertension and PIt counts, studies on patients with transjugular intrahepatic portosystemic shunt (TIPS) were undertaken. In one study, 55 TIPS patients were compared with 110 control patients to determine the effect of portal decompression on PIt counts pre- and post-procedure, over 12 months. The median Plt counts of patients post-TIPS increased by $19.7 \%$ $\left(104 \times 10^{9} / \mathrm{L}\right.$ to $\left.124.5 \times 10^{9} / \mathrm{L}\right)$, whereas the control Plt counts decreased by $17.1 \% .^{28}$ The greatest change in Plt counts occurred in the subgroup that had baseline TCP of $<100 \times 10^{9} / \mathrm{L}$. There were 20 TIPS patients and 43 control patients analyzed due to deaths or loss to follow-up. In that population, by month 12 post-TIPS, the median Plt counts increased by $36.8 \%$ and Plt counts increased by at least $25 \%$ above baseline in every patient in this group. The 
median increase in Plt count was by $26.5 \times 10^{9} / \mathrm{L}$, whereas in the moderate TCP group (PIt count $101-149 \times 10^{9} / \mathrm{L}$ ) there was only a $1 \times 10^{9} / \mathrm{L}$ increase. In the control group, the PIt counts decreased by $14.3 \%$. Also, there was an increase in PIt counts in patients that had a smaller decrease in portosystemic shunt gradient after TIPS ( $\geq 12 \mathrm{mmHg}$ ) compared to those that had a larger gradient difference $(<12 \mathrm{mmHg}) .^{28}$ Appropriate patient populations were excluded, limiting confounding factors for TCP. This was a prospective study with a control group, which many other studies have lacked. ${ }^{29,30}$ However, only patients with severe TCP showed significant improvement in PIt counts and no group reached normal counts. The data from the portocaval shunt gradients lead to conclusions opposite to that expected based on spleen size alone. These data support the conclusion that hypersplenism with sequestration cannot be the sole mechanism of TCP.

\section{Decreased production}

\section{Bone marrow suppression}

Viral bone marrow suppression has been hypothesized to be a mechanism of development of TCP in chronic HCV patients. However, data on bone marrow suppression by $\mathrm{HCV}$ is extremely limited. One study compared PIt counts in HCV patients before and 6 months after IFN therapy. They found that the post-treatment PIt counts increased by $25 \%$ relative to the baseline value $(p=0.027)$. Twelve of twenty-two who responded to IFN therapy with clearance of HCV showed an increase in PIt count. Yet, in the 10 patients whose HCV did not respond to IFN, PIt counts decreased by $25 \% .{ }^{18}$ The improvement in TCP after completion and cessation of IFN therapy could have been due to elimination of IFN-suppression of bone marrow or improvement in liver function, as described in a subsequent section, and not necessarily HCVinduced bone marrow suppression. A strength of the study is that cirrhosis and splenomegaly were excluded. This is an important distinction, as the authors controlled for hypersplenism and decreased TPO production as causes of TCP. However, although they measured TPO antibodies before IFN treatment of $\mathrm{HCV}$, they did not remeasure levels after treatment. TPO levels were higher prior to initiation of IFN, which was expected due to IFN-bone marrow suppression. It would have been helpful to have data on TPO levels after treatment of $\mathrm{HCV}$ with and without eradication of $\mathrm{HCV}$. Although this was a relatively small study, it does support the role of HCV-bone marrow suppression but requires further confirmation.

\section{Decreased TPO production}

In response to an increased demand for PIts, the number and size of megakaryocytes increase under the stimulation of TPO, a hematopoietic factor that regulates this response at various levels. The primary site of TPO mRNA and protein synthesis is the liver. Lesser amounts are found in the kidney, brain, and testes. There is no significant storage of TPO. It is synthesized and immediately released. With persistent TCP, TPO levels increase exponentially and reach a steady state. TPO levels increase within 24 hours after the onset of TCP and levels are inversely and exponentially proportional to the PIt count. Also, in the absence of Plts, there is little clearance of TPO by PIts, levels rise, bone marrow megakaryocytes are stimulated, and PIt production increases. TPO is cleared by attachment to Plts. ${ }^{31}$

As shown in Fig. 3, TPO levels are appropriately high in aplastic anemia and ITP (due to positive feedback). ${ }^{7,32}$ In the liver, as fibrosis advances to cirrhosis, liver mass decreases, resulting in levels of inappropriately low TPO. Adinolfi et al. ${ }^{27}$ studied 124 patients and compared Plt counts and TPO concentrations at different stages of fibrosis. The TPO levels were measured and compared in 54 of those patients. As shown in Fig. 4, the median TPO levels $(\mathrm{pg} / \mathrm{mL})$ per stage of fibrosis were reported as follows: stage $0-1: 58$, stage $2: 48$, stage $3: 36$, and stage $4: 27$. The median TPO levels of stage 3-4 fibrosis were significantly lower compared to stage $0-2(p<0.001)$.

One study sought to determine the effect of reducing hypersplenism by decreasing spleen size after splenic embolization, and improving synthetic liver function by liver transplantation on TCP. A total of 33 cirrhotic patients were studied, 24 of whom had cirrhosis due to HCV. Compared to the controls, the cirrhotic cohort had significantly lower TPO levels (median of $120.7 \mathrm{pg} / \mathrm{mL}$ compared to $756.4 \mathrm{pg} / \mathrm{mL}$ in non-cirrhotics) $(p<0.001) .{ }^{33}$ That population was then subdivided into two sub-cohorts of 22 patients who underwent splenic embolization and 11 who had liver transplants. In the splenic embolization sub-cohort whose spleen size decreased but had no change in liver mass, TPO levels increased by $94 \%$ and Plt counts increased by $84 \%$ at day 90 , respectively (TPO on day 0 was $153.7 \mathrm{pg} / \mathrm{mL}$ and 282.1 on day $90, p<0.05)$. PIt counts on day 0 were $50.5 \times 10^{9} / \mathrm{L}$ and $98 \times 10^{9} / \mathrm{L}$ on day $\left.\left.90, p<0.05\right)\right)^{33}$ This is consistent with decreased PIt sequestration. However, TPO levels unexpectedly increased after splenic-embolization. After liver transplantation, which decreased spleen size but increased liver mass, TPO levels increased by $288 \%$ and Plt counts increased by $175 \%$ at day 90 (TPO on day 0 was $43 \mathrm{pg} / \mathrm{mL}$ and 166.9 on day 90$)$. Plt counts on day 0 were $56 \times 10^{9} / \mathrm{L}$ and $154 \times 10^{9} / \mathrm{L}$ on day $90, p<0.05) .{ }^{33}$ With liver failure, TPO levels are inappropriately low prior to transplantation. After transplantation, spleen size is decreased, and the synthetic function of the liver increased. With normalization of PIt counts at 90 days, TPO levels were found to be appropriately increased from

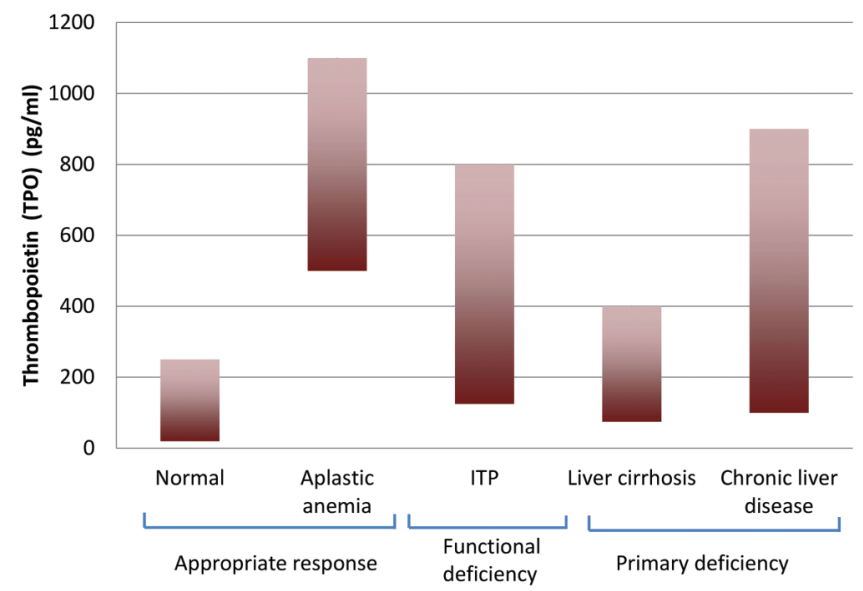

Fig. 3. Mean ranges of thrombocytopenia levels in various disease states: aplastic anemia, idiopathic thrombocytopenia purpura (ITP), liver cirrhosis, and chronic liver disease.

Modified from Giannini et al.7,32 
Rawi S. et al: TCP in chronic HCV: Mechanisms and treatment

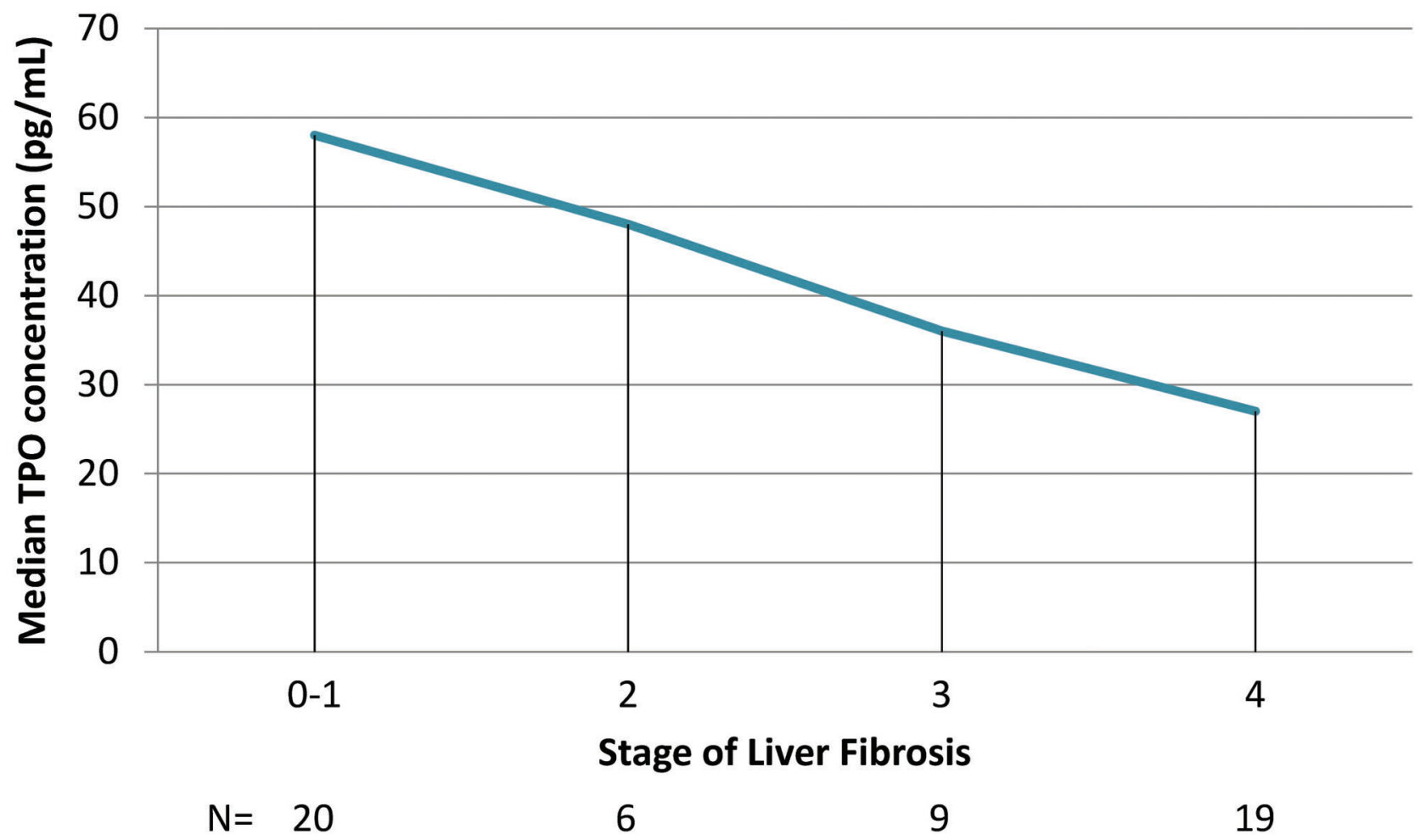

Fig. 4. Relationship between serum thrombocytopenia concentration and liver fibrosis.

Modified from Weksler et al. ${ }^{11}$ and Adinolfi et al. ${ }^{27}$

baseline. It would be helpful to have had a longer study period to determine if TPO levels returned to normal at later points after transplantation. In this study, it is difficult to conclude that decreased TPO production is the leading cause of TCP. However, it seems likely that the combination of decreased TPO production and splenic sequestration due to splenomegaly from portal hypertension are involved in the pathogenesis of TCP, rather than hypersplenism alone.

Eltrombopag, an oral TPO-receptor agonist, has been shown to increase Plt counts in hepatitis $C$ patients without reducing portal hypertension or splenomegaly. ${ }^{34}$ Eltrombopag interacts with the trans-membrane domain of the TPO receptor, activating JAK2/STAT signaling pathways and increasing proliferation and differentiation of human bone marrow progenitor cells into megakaryocytes. McHutchison et al. ${ }^{34}$ studied 45 chronic HCV cirrhotic patients and their median Plt counts over 112 days. The selection of patients was based on the following inclusion criteria: Plt count less than $70 \times 10^{9} / \mathrm{L}$, diagnosis of chronic $\mathrm{HCV}$, and evidence of compensated cirrhosis (through either liver biopsy, imaging, or endoscopic evidence of portal hypertension). Exclusion criteria included a history of thrombosis, pregnancy, or coinfection with hepatitis B or human immunodeficiency virus. Study patients received either varying doses of eltrombopag or placebo. If Plt counts increased above $70 \times 10^{9} / \mathrm{L}$, those individuals were eligible to be treated with IFN per investigator's discretion. It was found that as the IFN dose increased, PIt counts decreased. However, with eltrombopag treatment, counts never declined below the minimum threshold necessary for treatment. This study showed that eltrombopag can increase PIt counts allowing patients to be treated for longer periods of time and at higher IFN doses. The study was a multicenter, double-blinded, centrally randomized, intention-to-treat analysis, and placebo-controlled with an effective power for the primary endpoint of increasing baseline PIt count to $100 \times 10^{9} / \mathrm{L}$. During the study, eltrombopag was discontinued if Plts reached $200 \times 10^{9} / \mathrm{L}$ and was then reinitiated if they dropped again below $100 \times 10^{9} / \mathrm{L}$. Another point that supports the conclusions was that the controls' PIt counts never increased more than the treated groups, even after pegylated-IFN treatment. However, there were adverse events (62 events and 7 serious events reported), which can complicate the use of eltrombopag for chronic HCV. Overall, the study not only showed an effective treatment for TCP in chronic HCV, but also substantiated the pathophysiological mechanism of decreased TPO production leading to TCP in this population.

Sanjo et al. ${ }^{35}$ compared patients with chronic hepatitis, liver cirrhosis and controls. They concluded that their TPO levels were not significantly different. However, their PAIgG levels were elevated up to $144.6 \pm 113.6 \mathrm{ng} / 10^{7}$ cells (compared to the controls which had levels of $18.9 \pm 2.5 \mathrm{ng} / 10^{7}$ cells, $p<0.001)$, thus supporting autoimmune mechanisms of TCP. However, they also reported that the liver volumes between the three cohorts were not significantly different, suggesting that the healthy liver mass producing TPO was not significantly different as well. This suggested that low TPO was not a major cause of TCP in this population, and that TPO deficiency cannot be the sole factor in the development of TCP. However, this conclusion is not completely justified because changes in liver volumes were not measured. If there were no differences in liver volume between the 
controls and the chronic hepatitis/cirrhosis groups, that would suggest only patients with low stages of fibrosis were enrolled in this study.

\section{Treatment}

At present, four TPO-agonists (eltrombopag, romiplostim, avatrombopag and lusutrombopag) have been approved for HCV-related TCP. ${ }^{34,36-39}$ Eltrombopag, as mentioned above, is an orally active TPO-agonist that increases differentiation of bone marrow progenitor cells leading to production of megakaryocytes. ${ }^{34,36,40}$ Romiplostim is a polypeptide composed of four TPO mimetic peptides that dimerize TPO, leading to increase Plt production. ${ }^{36,37}$ The two newest medications, avatrombopag and lusutrombopag, are both non-peptide oral TPO agonists. ${ }^{39}$ By July 2019, the USA's Federal Drug Administration and European Medicines Agency had approved all medications, except romiplostim, for treatment of TCP in chronic HCV patients. ${ }^{41-46}$ Eltrombopag and romiplostim were additionally approved for ITP. ${ }^{41,42}$ Studies on the use of eltrombopag, including large phase 3 randomized, controlled, open-labeled studies called ENABLE-1 and ENABLE2 , have primarily focused on improving PIt counts in order to improve ribavirin and IFN HCV therapy. ${ }^{34,47,48}$ The effect of eltrombopag on PIt count was studied in a phase 1 trial; however, neither chronic HCV nor thrombocytopenic populations were included. ${ }^{40}$ Eltrombopag shows potential benefit for invasive procedures or surgery in patients with TCP due to liver disease.

Romiplostim, on the other hand, was studied over 90 days preoperatively in 35 patients with chronic liver disease and TCP secondary to HCV infection. ${ }^{37}$ The goal of that study was to improve PIt counts greater than $70 \times 10^{9} / \mathrm{L}$ in order to allow the patient to undergo surgical interventions. The patients in that study were refractory to other standard treatment (e.g., PIt transfusion, folic acid, antioxidants), non-splenectomized, and scheduled for non-emergent surgical procedures. Mean Plt counts increased more than three-fold over baseline after 3 weeks of therapy, and remained at least one and a half times above the baseline even 2 months after discontinuation of the drug. Plt counts peaked at days 18 to 39, with a maximum level of $99 \times 10^{9} / \mathrm{L}$. However, the study was a small, single-center study in Egypt, limiting extrapolation of data from this specific demographic to others around the world. Another disadvantage of the reported data is that it took weeks of treatment to achieve a positive change. The study did show that all the patients who failed other treatments responded to romiplastin, and only two patients failed to meet their target Plt count. There were also no reported adverse effects or serious events within 60 days of the surgeries. ${ }^{37}$ There appears to be great potential in the use of TPO-agonists for increasing Plt counts for invasive procedures, and this led to two large randomized control studies that tested the use of avatrombopag and lusutrombopag prior to elective procedures. ${ }^{39}$ Avatrombopag and lusutrombopag have been studied for the primary purpose of reducing preprocedural Plt transfusions. Up to $88 \%$ and $65 \%$ of patients in each trial reached this goal, respectively. Their secondary endpoint was to determine whether Plt counts increased to above $50 \times 10^{9} / \mathrm{L}$, which was also achieved in up to $69 \%$ and $68 \%$ of patients on procedure day, respectively. ${ }^{49,50} \mathrm{~A}$ strength of that study was the patient population and placebo group. A weakness of the study included restriction to severe TCP (PIt counts $<50 \times 10^{9} / \mathrm{L}$ ), which was also the PIt transfusion criterion. Further studies on the use of these medications for chronic HCV-TCP population at moderate levels as well as severe levels for chronic elevation of Plt counts are needed. Furthermore, long-term benefit and risks need to be determined.

To evaluate possible virus-induced bone marrow suppression, Moussa and Mowafy ${ }^{37}$ performed bone marrow biopsies on patients with HCV who were due for surgery. Samples were taken on days 0 and 90 . On day 0 , no patient had hypocellular bone marrow, thus indirectly showing that HCV did not cause decreased production of megakaryocytes. On day 90, there were five patients who began with a hypercellular state and changed to a normocellular state. Four patients changed in the opposite direction. Again, no patient had hypocellular marrow, which again showed that neither HCV nor romiplostim had significant effect on bone marrow production at this point of therapy.

Other TPO-agonists have been studied including interleukin 11, AMG-531 and PEG-TPOmp, non-peptide compounds like AKR-501, monoclonal antibodies, synthetic recombinant human TPO, pegylated-recombinant human megakaryocyte growth and development factor, recombinant human erythropoietin, danazol, and L-carnitine. ${ }^{36,51-53}$ There are insufficient data to draw meaningful conclusions, and more clinical trials are needed.

\section{Discussion}

Currently, although several TPO-agonists are approved by the Federal Drug Administration and available, not all societal guidelines have recommended their routine use. For example, neither the American Association for the Study of Liver Diseases nor the Infectious Diseases Society of America have specific HCV TCP guidelines. However, the 2019 American Gastroenterological Association Clinical Update states, as part of their best practice guidelines, that TPO receptor agonists should be used for treatment of TCP in cirrhotic patients if the patient has sufficient time for the agent to be effective. ${ }^{54}$ In addition, there is no recommendation for routine measurement of TPO before or during treatment.

It would seem to be beneficial to check TPO levels prior to use of a TPO-agonist to determine if the patient actually requires further megakaryocyte stimulation or not. It would be ineffective and wasteful to treat patients who already have high levels of TPO, and could expose patients unnecessarily to side effects.

TPO-agonists have reversible side effects as well as longterm effects. It is important to note that there have been studies that have associated a high incidence of thromboembolic events, including portal vein thrombosis, with the use of eltrombopag. ${ }^{55,56}$ There were initial concerns for bone marrow fibrosis and increased risk of malignancy due to the constant stimulation of stem cells/megakaryocytes. However, after 10 years of observation of eltrombopag and romiplostim, this has not been found. ${ }^{56}$ On the other hand, rebound TCP after abrupt discontinuation of romiplostim has been reported ${ }^{31}$ as well as cataracts and aminotransferase elevations with eltrombopag use. ${ }^{56}$ Due to these side effects and limitations, other TPO-agonists, avatrombopag and lusutrombopag, have been developed.

At this time, the use of these new medications must be limited to elective procedures, as they require at least 5 to 7 days to increase Plt counts. Plt counts also quickly decline after initial dosing, ${ }^{49,50}$ so patients would require intermittent 
or continuous use of the medication for long-term elevation of Plt counts. The risks of long-term elevation of PIt counts in this population are not known. On the other hand, the benefits of using these drugs to avoid surgery in high-risk surgical patients with low PIt counts can be substantial. In addition, such therapy could decrease the number of pre-procedural transfusions, thus reducing cost, risk of allergic reactions, and acquired infections. Data on successful treatment of TCP prior to surgery in patients with liver disease are convincing. Future studies will be needed to determine the long-term effects and the expansion of the use of avatrombopag and lusutrombopag.

\section{Conclusions}

The pathogenesis of TCP in chronic HCV patients is multifactorial and multifaceted. Four general pathogenic mechanisms have been proposed. Two mechanisms deal with increased destruction which include autoimmune antibody Plt destruction and hypersplenism with sequestration. Two mechanisms involve decreased production, including virusinduced bone marrow suppression and decreased TPO production. Of these, virus-induced bone marrow suppression has the least support. There are data supporting the coexistence of several mechanisms causing TCP due to chronic $\mathrm{HCV}$ and cirrhosis. More research is needed to better understand the factors involved in development of TCP in patients with liver disease and for the production of other agents with differing mechanisms of action in the treatment of TCP.

\section{Acknowledgements}

The support of the Herman Lopata Chair in Hepatitis is gratefully acknowledged.

\section{Funding}

None to declare.

\section{Conflict of interest}

The authors have no conflict of interests related to this publication.

\section{Author contributions}

Wrote the manuscript and prepared the figures (SR), proposed the idea for the review and critically revised the manuscript (GYW).

\section{References}

[1] National Heart Lung and Blood Institute. Thrombocytopenia. Available from: https://www.nhlbi.nih.gov/health-topics/thrombocytopenia.

[2] Siegel RS, Rae JL, Barth S, Coleman RE, Reba RC, Kurlander R, et al. Platelet survival and turnover: important factors in predicting response to splenectomy in immune thrombocytopenic purpura. Am J Hematol 1989;30:206212. doi: 10.1002/ajh.2830300404.

[3] World Health Organization. Hepatitis C. Available from: https://www.who. int/news-room/fact-sheets/detail/hepatitis-c.

[4] American Liver Foundation. Liver disease statistics. Available from: https: //liverfoundation.org/liver-disease-statistics/.

[5] Louie KS, Micallef JM, Pimenta JM, Forssen UM. Prevalence of thrombocytopenia among patients with chronic hepatitis C: a systematic review. J Viral Hepat 2011;18:1-7. doi: 10.1111/j.1365-2893.2010.01366.x.
[6] Giannini EG. Review article: thrombocytopenia in chronic liver disease and pharmacologic treatment options. Aliment Pharmacol Ther 2006;23:10551065. doi: 10.1111/j.1365-2036.2006.02889.x.

[7] Giannini EG, Savarino V. Epidemiology of thrombocytopenia in patients with chronic hepatitis C: more than meets the eye. J Viral Hepat 2011;18:8-10. doi: $10.1111 / \mathrm{j} .1365-2893.2010 .01368 . x$.

[8] Arnold DM. Bleeding complications in immune thrombocytopenia. Hematology Am Soc Hematol Educ Program 2015;2015:237-242. doi: 10. 1182/asheducation-2015.1.237.

[9] Lv Y, Lau WY, Li Y, Deng J, Han X, Gong X, et al. Hypersplenism: History and current status. Exp Ther Med 2016;12:2377-2382. doi: 10.3892/etm.2016. 3683.

[10] Pawlotsky JM, Ben Yahia M, Andre C, Voisin MC, Intrator L, Roudot-Thoraval $\mathrm{F}$, et al. Immunological disorders in $\mathrm{C}$ virus chronic active hepatitis: a prospective case-control study. Hepatology 1994;19:841-848. doi: 10. 1002/hep.1840190407.

[11] Weksler BB. Review article: the pathophysiology of thrombocytopenia in hepatitis $C$ virus infection and chronic liver disease. Aliment Pharmacol Ther 2007;26 Suppl 1:13-19. doi: 10.1111/j.1365-2036.2007.03512.x.

[12] Pereira J, Accatino L, Alfaro J, Brahm J, Hidalgo P, Mezzano D. Platelet autoantibodies in patients with chronic liver disease. Am J Hematol 1995;50: 173-178. doi: 10.1002/ajh.2830500305.

[13] Aoki Y, Hirai K, Tanikawa K. Mechanism of thrombocytopenia in liver cirrhosis: kinetics of indium-111 tropolone labelled platelets. Eur J Nucl Med 1993; 20:123-129. doi: 10.1007/bf00168872.

[14] Pradella P, Bonetto S, Turchetto S, Uxa L, Comar C, Zorat F, et al. Platelet production and destruction in liver cirrhosis. J Hepatol 2011;54:894-900. doi: 10.1016/j.jhep.2010.08.018.

[15] Liebman HA. Viral-associated immune thrombocytopenic purpura. Hematology Am Soc Hematol Educ Program 2008:212-218. doi: 10.1182/asheducation-2008.1.212

[16] Pockros PJ, Duchini A, McMillan R, Nyberg LM, McHutchison J, Viernes E. Immune thrombocytopenic purpura in patients with chronic hepatitis $\mathrm{C}$ virus infection. Am J Gastroenterol 2002;97:2040-2045. doi: 10.1111/j. 1572-0241.2002.05845.x.

[17] Nagamine T, Ohtuka T, Takehara K, Arai T, Takagi H, Mori M. Thrombocytopenia associated with hepatitis C viral infection. J Hepatol 1996;24:135140. doi: $10.1016 / \mathrm{s} 0168-8278(96) 80021-3$.

[18] Iga D, Tomimatsu M, Endo H, Ohkawa S, Yamada O. Improvement of thrombocytopenia with disappearance of HCV RNA in patients treated by interferon-alpha therapy: possible etiology of HCV-associated immune thrombocytopenia. Eur J Haematol 2005;75:417-423. doi: 10.1111/j. 1600-0609.2005.00524.x.

[19] Honma Y, Shibata M, Hayashi T, Kusanaga M, Ogino N, Minami S, et al. Effect of direct-acting antivirals on platelet-associated immunoglobulin $G$ and thrombocytopenia in hepatitis $C$ virus-related chronic liver disease. Liver Int 2019;39:1641-1651. doi: 10.1111/liv.14120.

[20] Taliani G, Duca F, Clementi C, De Bac C. Platelet-associated immunoglobulin $\mathrm{G}$, thrombocytopenia and response to interferon treatment in chronic hepatitis C. J Hepatol 1996;25:999. doi: 10.1016/s0168-8278(96)80309-6.

[21] Aster RH. Pooling of platelets in the spleen: role in the pathogenesis of "hypersplenic" thrombocytopenia. J Clin Invest 1966;45:645-657. doi: 10. 1172/JCI105380.

[22] Penny R, Rozenberg MC, Firkin BG. The splenic platelet pool. Blood 1966;27: 1-16.doi: 10.1182/blood.V27.1.1.1

[23] McCormick PA, Murphy KM. Splenomegaly, hypersplenism and coagulation abnormalities in liver disease. Baillieres Best Pract Res Clin Gastroenterol 2000;14:1009-1031. doi: 10.1053/bega.2000.0144.

[24] Noguchi H, Hirai K, Aoki Y, Sakata K, Tanikawa K. Changes in platelet kinetics after a partial splenic arterial embolization in cirrhotic patients with hypersplenism. Hepatology 1995;22:1682-1688. doi: 10.1002/hep.1840220611.

[25] Hill-Zobel RL, McCandless B, Kang SA, Chikkappa G, Tsan MF. Organ distribution and fate of human platelets: studies of asplenic and splenomegalic patients. Am J Hematol 1986;23:231-238. doi: 10.1002/ajh.2830230307.

[26] Sekiguchi T, Nagamine T, Takagi $\mathrm{H}$, Mori M. Autoimmune thrombocytopenia in response to splenectomy in cirrhotic patients with accompanying hepatitis $\mathrm{C}$. World J Gastroenterol 2006;12:1205-1210. doi: 10.3748/wjg.v12.i8.1205.

[27] Adinolfi LE, Giordano MG, Andreana A, Tripodi MF, Utili R, Cesaro G, et al. Hepatic fibrosis plays a central role in the pathogenesis of thrombocytopenia in patients with chronic viral hepatitis. $\mathrm{Br}$ J Haematol 2001;113:590-595. doi: $10.1046 / j .1365-2141.2001 .02824 . x$.

[28] Gschwantler M, Vavrik J, Gebauer A, Kriwanek S, Schrutka-Kölbl C, Fleischer $\mathrm{J}$, et al. Course of platelet counts in cirrhotic patients after implantation of a transjugular intrahepatic portosystemic shunt-a prospective, controlled study. J Hepatol 1999;30:254-259. doi: 10.1016/s0168-8278(99)80071-3.

[29] Jalan R, Redhead DN, Allan PL, Hayes PC. Prospective evaluation of haematological alterations following the transjugular intrahepatic portosystemic stent-shunt (TIPSS). Eur J Gastroenterol Hepatol 1996;8:381-385. doi: 10.1097/00042737-199604000-00017. 
[30] Sanyal AJ, Freedman AM, Purdum PP, Shiffman ML, Luketic VA. The hematologic consequences of transjugular intrahepatic portosystemic shunts. Hepatology 1996;23:32-39. doi: 10.1002/hep.510230105.

[31] Kuter DJ. Thrombopoietin: biology, clinical applications, role in the donor setting. J Clin Apher 1996;11:149-159.doi: 10.1002/(SICI)1098-1101 (1996)11:3<149::AID-JCA6>3.0.CO;2-B

[32] Nichol JL. Endogenous TPO (eTPO) levels in health and disease: possible clues for therapeutic intervention. Stem Cells 1998;16 Suppl 2:165-175. doi: 10.1002/stem.5530160719.

[33] Rios R, Sangro B, Herrero I, Quiroga J, Prieto J. The role of thrombopoietin in the thrombocytopenia of patients with liver cirrhosis. Am J Gastroenterol 2005;100:1311-1316. doi: 10.1111/j.1572-0241.2005.41543.x.

[34] McHutchison JG, Dusheiko G, Shiffman ML, Rodriguez-Torres M, Sigal S, Bourliere $\mathrm{M}$, et al. Eltrombopag for thrombocytopenia in patients with cirrhosis associated with hepatitis C. N Engl J Med 2007;357:2227-2236. doi: 10. 1056/NEJMoa073255

[35] Sanjo A, Satoi J, Ohnishi A, Maruno J, Fukata M, Suzuki N. Role of elevated platelet-associated immunoglobulin $\mathrm{G}$ and hypersplenism in thrombocytopenia of chronic liver diseases. J Gastroenterol Hepatol 2003;18:638-644. doi: 10.1046/j.1440-1746.2003.03026.x

[36] Dahal S, Upadhyay S, Banjade R, Dhakal P, Khanal N, Bhatt VR. Thrombocytopenia in patients with chronic hepatitis C virus infection. Mediterr J Hematol Infect Dis 2017;9:e2017019. doi: 10.4084/MJHID.2017.019.

[37] Moussa MM, Mowafy N. Preoperative use of romiplostim in thrombocytopenic patients with chronic hepatitis $\mathrm{C}$ and liver cirrhosis. J Gastroenterol Hepatol 2013;28:335-341. doi: 10.1111/j.1440-1746.2012.07246.x.

[38] Voican CS, Naveau S, Perlemuter G. Successful antiviral therapy for hepatitis $C$ virus-induced cirrhosis after an increase in the platelet count with romiplostim: two case reports. Eur J Gastroenterol Hepatol 2012;24:1455-1458. doi: 10.1097/MEG.0b013e328357d5f2.

[39] Nilles KM, Caldwell SH, Flamm SL. Thrombocytopenia and procedural prophylaxis in the era of thrombopoietin receptor Agonists. Hepatol Commun 2019;3:1423-1434. doi: 10.1002/hep4.1423.

[40] Jenkins JM, Williams D, Deng Y, Uhl J, Kitchen V, Collins D, et al. Phase 1 clinical study of eltrombopag, an oral, nonpeptide thrombopoietin receptor agonist. Blood 2007;109:4739-4741. doi: 10.1182/blood-2006-11057968.

[41] U.S. Food \& Drug Administration. Promacta (eltrombopag) information. Available from: https://www.fda.gov/drugs/postmarket-drug-safety-information-patients-and-providers/promacta-eltrombopag-information.

[42] U.S. Food \& Drug Administration. Nplate (romiplostim) information. Available from: https://www.fda.gov/drugs/postmarket-drug-safety-information-patients-and-providers/nplate-romiplostim-information.

[43] U.S. Food \& Drug Administration. FDA approves avatrombopag for thrombocytopenia in adults with chronic liver disease. Available from: https://www. fda.gov/drugs/resources-information-approved-drugs/fda-approves-avatrombopag-thrombocytopenia-adults-chronic-liver-disease.

[44] U.S. Food \& Drug Administration. FDA approves lusutrombopag for thrombocytopenia in adults with chronic liver disease. Available from: https: //www.fda.gov/drugs/resources-information-approved-drugs/fda-approves-lusutrombopag-thrombocytopenia-adults-chronic-liver-disease.

[45] European Medicines Agency. Nplate. Available from: https://www.ema. europa.eu/en/medicines/human/EPAR/nplate.

[46] European Medicines Agency. Revolade. Available from: https://www.ema. europa.eu/en/medicines/human/EPAR/revolade.

[47] Afdhal NH, Dusheiko GM, Giannini EG, Chen PJ, Han KH, Mohsin A, et al. Eltrombopag increases platelet numbers in thrombocytopenic patients with HCV infection and cirrhosis, allowing for effective antiviral therapy. Gastroenterology 2014;146:442-452.e1. doi: 10.1053/j.gastro.2013.10.012.

[48] Mihăilă RG, Cipăian RC. Eltrombopag in chronic hepatitis C. World J Gastroenterol 2014;20:12517-12521. doi: 10.3748/wjg.v20.i35.12517.

[49] Terrault N, Chen YC, Izumi N, Kayali Z, Mitrut P, Tak WY, et al. Avatrombopag before procedures reduces need for platelet transfusion in patients with chronic liver disease and thrombocytopenia. Gastroenterology 2018;155: 705-718. doi: 10.1053/j.gastro.2018.05.025.

[50] Peck-Radosavljevic M, Simon K, Iacobellis A, Hassanein T, Kayali Z, Tran A, et al. Lusutrombopag for the treatment of thrombocytopenia in patients with chronic liver disease undergoing invasive procedures (L-PLUS 2). Hepatology 2019;70:1336-1348. doi: 10.1002/hep.30561.

[51] de Serres M, Ellis B, Dillberger JE, Rudolph SK, Hutchins JT, Boytos CM, et al. Immunogenicity of thrombopoietin mimetic peptide GW395058 in BALB/C mice and New Zealand white rabbits: evaluation of the potential for thrombopoietin neutralizing antibody production in man. Stem Cells 1999;17:203209. doi: 10.1002/stem.170203.

[52] Dower WJ, Cwirla SE, Balasubramanian P, Schatz PJ, Baccanari DP, Barrett RW. Peptide agonists of the thrombopoietin receptor. Stem Cells 1998;16 Suppl 2:21-29. doi: 10.1002/stem.5530160705.

[53] Kaushansky K. Hematopoietic growth factor mimetics. Ann N Y Acad Sci 2006;938:131-138. doi: 10.1111/j.1749-6632.2001.tb03582.x

[54] O'Leary JG, Greenberg CS, Patton HM, Caldwell SH. AGA clinical practice update: Coagulation in cirrhosis. Gastroenterology 2019;157:34-43.e1. doi: 10.1053/j.gastro.2019.03.070.

[55] Afdhal NH, Giannini EG, Tayyab G, Mohsin A, Lee JW, Andriulli A, et al. Eltrombopag before procedures in patients with cirrhosis and thrombocytopenia. $\mathrm{N}$ Engl J Med 2012;367:716-724. doi: 10.1056/NEJMoa1110709.

[56] Ghanima W, Cooper N, Rodeghiero F, Godeau B, Bussel JB. Thrombopoietin receptor agonists: ten years later. Haematologica $2019 ; 104: 1112-1123$. doi: $10.3324 /$ haematol.2018.212845. 